\title{
Studies of animal development using a 4D-microscopy sytem
}

\section{Andreas Hejnol, Ralf Schnabel}

Technische Universität Braunschweig, Institut für Genetik, Spielmannstr. 7, 38106 Braunschweig, Germany

The development of an organism is composed of processes occuring in space and time. To analyze a 4-dimensional process, an appropriate method should be used, that is adapted to this nature of an embryogenesis. We present her a sophisticated microscopy method, developed to execute analysis of cell lineage mutant of the nematode Caenorhabditis elegans. This 4D-microscopy (3Dtimelapse) enables us to record the whole development of an embryo and to analyze the main cell lineage events, like cleavage, cell migration, cell death (apoptosis) and cell differentiation during development in detail. The 4D-microscopy system is composed out of three main parts, a motorized microscope, a trigger-software and a database that facilitates the analysis of the recordings. We used only Nomarski Optics to visualize the cell events in every level. We adopted this 4Dmicroscopy technique to other embryos, what made it possible to study the cell lineage of small embryos. Our method fills a gap in the study of the development of many diverse organisms, which are not accessible with common labeling techniques of single blastomeres with fluorescent dyes. The use of this technique to describe and reinvestigate the development of organisms like tardigrades, acoels, rotifers, gastrotrichs opens new insights on the evolution of developmental processes and the phylogenetic relationships between this taxa. 
